\title{
Review of: "Volumetric Tomographic 3D Bioprinting of Heterocellular Bone-like Tissues in Seconds"
}

\author{
Ruijian Yan ${ }^{1}$ \\ 1 Zhejiang University
}

Potential competing interests: The author(s) declared that no potential competing interests exist.

\section{Comments to the author}

This paper proposed a volumetric tomographic 3D printing technique and its related model for angiogenesis. However, so-called in seconds speed has not been addressed as the title. Actually, it is just a photofabrication method which has been reported commonly, and it is not so innovative. Meanwhile, the osteogenic differentiation of the hMSC and co-culture of the HUVECs was not verified adequately. Thus, there are still several major concerns that should be addressed before publication.

1. P15, line14. The lap group at different concentrations has diverse cell morphology, which may be due to its physical structure. A more detailed characterization should be performed to confirm it?

2. P15, the author stated that the cells in the high-concentration lap group are difficult to take nutrients from the surrounding matrix due to the physical structure. Are the high concentration group cells delayed in the formation of ossification? Is the osteogenic differentiation time of the cells of all groups different? 3. P16, line1. The author stated that the expression of osteogenic genes was not found in HUVECs in culture alone, and it had no effect on the total expression of osteogenic genes when they were co-cultured. But how to exclude the effect of hMSC on the expression of osteogenic genes in HUVECs when cocultured? 4. Figure 4. The duration of osteogenic gene expression is short, and its expression of monoculture is higher than the co-culture. However, the expression of osteogenic gene in co-culture is higher than the monoculture in long term. Is such phenomenon related the matrix used in this work? Does this phenomenon also exist in ordinary culture media?

5. P17, line18. The author claimed that these findings imply the temporal dynamics of gene expression in $3 D$ osteogenesis as well as the significant role of juxta- and/or paracrine signaling in cell activity. But the osteogenic-related genes expression in this paper do not reflect the specific role of HUVECs?

6. P18, line1. The author claimed that ALP activity in 3D was not matched to the ALPL gene expression patterns and rather stable expressed in both, mono- and co-culture, during the whole observation time. The differences in gene expression and enzyme activity between monoculture and co-cultures were also found in 2D cultures. Under the 2D environment, what are the specific conditions of ALP activity and ALPL gene expression in monoculture and co-culture conditions.

7. The author claimed that we observed the effects of co-cultures on osteogenic differentiation being less prominent in 2D cultures compared to 3D. The results of related experiment are descriptive. More details 
should be provided. In the 2D environment, which indicators are measured to reach this conclusion?

8. Figure $4 \mathrm{~d}$. The author claimed that the compressive moduli of the gel constructs increased from about 6 $\mathrm{kPa}(d 1)$ to $13 \mathrm{kPa}(d 7)$ and $46 \mathrm{kPa}(d 28)$, with an 8-fold increase between day 1 and 28. In monoculture circumstance, how does the compressive moduli of the substrate change over time, and is there a significant difference between them? 